\title{
Impact of complex segmentectomies by video-assisted thoracic surgery on peri-operative outcomes
}

\author{
Benoît Bédat ${ }^{1}$, Etienne Abdelnour-Berchtold ${ }^{2}$, Thortsen Krueger ${ }^{2}$, Jean Yannis Perentes ${ }^{2}$, \\ Matthieu Zellweger ${ }^{2}$, Frédéric Triponez ${ }^{1}$, Wolfram Karenovics ${ }^{1}$, Michel Gonzalez ${ }^{2}$ \\ ${ }^{1}$ Division of Thoracic and Endocrine Surgery, University Hospitals of Geneva, Geneva, Switzerland; ${ }^{2}$ Service of Thoracic Surgery, University \\ Hospital of Lausanne, Lausanne, Switzerland \\ Contributions: (I) Conception and design: M Gonzalez, B Bédat, W Karenovics; (II) Administrative support: M Gonzalez; (III) Provision of study \\ materials or patients: M Gonzalez, W Karenovics; (IV) Collection and assembly of data: M Gonzalez, B Bédat, W Karenovics; (V) Data analysis \\ and interpretation: M Gonzalez, B Bédat, W Karenovics, E Abdelnour-Berchtold; (VI) Manuscript writing: All authors; (VII) Final approval of \\ manuscript: All authors. \\ Correspondence to: Michel Gonzalez, MD. Service of Thoracic Surgery, University Hospital of Lausanne, University of Lausanne, Rue du Bugnon 46, \\ 1011-Lausanne, Switzerland. Email: michel.gonzalez@chuv.ch.
}

Background: Pulmonary segmentectomies are generally classified into simple (tri-segmentectomy or lingulectomy as well as apical or basilar segmentectomy) and complex (individual or bi-segmentectomy of the upper, middle and lower lobes). Complex segmentectomies are technically feasible by video-assisted thoracic surgery (VATS) but remain challenging, and reports on post-operative outcomes are scarce. This study analyzes the differences between simple and complex VATS segmentectomy in terms of peri- and postoperative outcomes.

Methods: We retrospectively reviewed records of all patients who underwent anatomical pulmonary segmentectomy by VATS from 2014 to 2018 in two university hospitals.

Results: A total of 232 patients (114 men; median age 67 years; range, 29-87 years) underwent VATS segmentectomy for primary lung cancer $(n=177)$, metastases $(n=26)$ and benign lesions $(n=29)$. The overall 30 -day mortality and morbidity rates were $0.8 \%$ and $29.7 \%$, respectively. The re-operation rate was $4.7 \%$. Complex segmentectomy was realized in 111 patients including 86 (77.5\%) upper lobe segmentectomies and $44(39.6 \%)$ bi-segmentectomies. There was no statistical difference between complex and simple segmentectomy in terms of operative time (145 vs. $143 \mathrm{~min}$, respectively; $\mathrm{P}=0.79)$ and chest tube duration [median: 1 (range, 0-33) vs. 2 (range, 1-19) days, respectively; $\mathrm{P}=0.95$ ]. Post-operative overall complication rates were similar for both groups $(30 \%$ vs. 30\%, respectively; $\mathrm{P}=0.99)$ and were not correlated with the type of segmentectomy. However, complex segmentectomy patients had a shorter length of hospitalization compared to simple segmentectomy patients [median: 5 (range, 1-36) vs. 7 (range, 2-31) days; $\mathrm{P}=0.026$ ]. Interestingly, complex segmentectomies were realized most frequently 2 years after implementation of VATS segmentectomy ( $23 \%$ vs. $77 \% ; \mathrm{P}=0.01)$.

Conclusions: In comparison with simple segmentectomy, complex segmentectomy by VATS seems to present similar post-operative complication rates. Learning curve and progressive increase in acceptance by surgeons seem to be key elements for successful implementation of complex segmentectomies and could explain the shorter length of stay we observed.

Keywords: Video-assisted thoracic surgery (VATS); segmentectomy; post-operative complication; lung cancer

Submitted Jun 07, 2019. Accepted for publication Aug 27, 2019.

doi: $10.21037 /$ jtd.2019.10.07

View this article at: http://dx.doi.org/10.21037/jtd.2019.10.07

(C) Journal of Thoracic Disease. All rights reserved. 


\section{Introduction}

Pulmonary segmentectomy is a parenchyma-sparing technique that is now currently proposed for diagnosis of centrally located nodules or for definitive treatment of metastases or early stage non-small cell lung cancer (NSCLC) in selected patients. Many retrospective studies have suggested that pulmonary segmentectomy can achieve recurrence rates and survival rates comparable to those of lobectomy in patients with small (diameter $<2 \mathrm{~cm}$ ), peripheral tumors when adequate surgical margins are achieved and lymph node are dissected and free of tumor (1-6). Nevertheless, pulmonary segmentectomy is a technically challenging procedure that is now performed by video-assisted thoracic surgery (VATS) (7). The large anatomical variations of each segment make this operation arduous since individual dissection of segmental bronchovascular structures and identification of the intersegmental planes are necessary to avoid incomplete resections and post-operative complications. Recently, we have reported on our initial experience and demonstrated that VATS segmentectomies result in favorable clinical outcomes with acceptable morbidity (8).

From a technical point of view, pulmonary segmentectomies can be classified into simple and complex procedures, based on the necessity to separate one or multiple intersegmental planes $(9,10)$. Simple segmentectomies (SS) involve one linear intersegmental plane and include segmentectomies of the left upper lobe (tri-segmentectomy or lingulectomy), apical segmentectomies (S6), and basal segmentectomies (S7-10). These are generally well standardized procedures, regularly performed by VATS and not prone to particular difficulties pertaining to anatomical variations or identification of intersegmental planes. On the other hand, complex segmentectomies (CS) involve several, or intricate intersegmental planes to separate segments and include individual or bi-segmentectomies of the upper lobe, middle lobe, or of the basal segments. In CS, bronchovascular structures are located more deeply in the lung parenchyma and are subject to wide individual anatomical variations (7). In addition, the VATS approach makes the procedure more challenging, possibly leading to potentially catastrophic complications or insufficient surgical margins. Few studies have reported the peri- and post-operative clinical outcomes of CS, and very few of them specially describe this procedure performed by VATS (10).

The aim of the study was to analyze the differences between simple and complex VATS segmentectomies in terms of peri- and post-operative outcomes in two university hospitals.

\section{Methods}

\section{Patients}

We retrospectively reviewed the records of all patients who underwent anatomical pulmonary segmentectomies by VATS from January 2014 to May 2018 at the University Hospitals of Geneva and Lausanne in Switzerland. The Local Ethics Committee approved the study and individual consent was waived (Referral number: 2018-00179). This study was reported according to the STROBE criteria for observational studies (Table S1). We excluded patients whose procedure required conversion to open thoracotomy and patients who had a completion lobectomy in case of incomplete resection or lymph node involvement on frozen section for the post-operative outcome analysis.

Simple segmentectomies were performed by surgeons with an experience of more than 100 VATS lobectomies. CS were performed or supervised by one surgeon in each hospital (M Gonzalez and W Karenovics). In NSCLC patients, positron emission tomography-computed tomography (PET-CT) imaging was performed to exclude lymph node involvement, as well as routine brain magnetic resonance imaging and pulmonary function testing. Endobronchial ultrasound (EBUS) was performed prior surgery in case of suspicion of mediastinal lymph node involvement. All patients were presented to a multidisciplinary board. In patients fit enough to tolerate a lobectomy, intentional VATS segmentectomy was proposed for tumors with a diameter of $<2 \mathrm{~cm}$ and a peripheral location well in a specific segment. In patients unfit for lobectomy, segmentectomy was proposed even for lesions of greater diameter. Staging was determined according to the $8^{\text {th }}$ TNM Classification of lung cancer.

\section{Surgery}

VATS segmentectomy was performed under general anesthesia with lung isolation by double lumen intubation. Surgical resection was undertaken using a standardized three-port approach (utility incision of 3 to $4 \mathrm{~cm}$ in the fourth intercostal space, one camera port in the seventh intercostal space anteriorly and a third posterior port for instrumentation). Segmentectomy was performed with 
individual dissection of the segmental bronchus, arteries and veins. All bronchovascular structures were transected using endoscopic staplers or energy device. In cases of NSCLC, systematic dissection of hilar and mediastinal lymph nodes was carried out. Frozen section was performed for suspicious hilar lymph nodes and completion lobectomy was undertaken if positive. Once the intersegmental plane was identified by inflation/deflation technique or using indocyanine green (ICG) fluorescence, staplers were used to separate the intersegmental planes. All surgical specimens were extracted in a protective bag to prevent chest wall seeding of malignant disease.

\section{Measurements and outcomes}

All data was prospectively collected from our shared database. Individual medical records were then retrospectively reviewed and analyzed with respect to: patient demographics; pulmonary function; type of segmentectomy; histologic findings; size and localization of the lesion; operative time; surgical outcome; post-operative morbidity and mortality.

CS group included individual segmentectomies of the upper or lower lobes or combined bi-segmentectomies of the same lobe $(\mathrm{S} 1+2, \mathrm{~S} 1+3, \mathrm{~S} 9+10, \mathrm{~S} 8+9)$. SS group included upper lobe trisegmentectomies $(\mathrm{S} 1+2+3)$, lingulectomies, S6 segmentectomy of the lower lobe and basal segmentectomies $[\mathrm{S}(7)+8+9+10]$.

The primary endpoint was 30-day cardio-pulmonary complication rate and included: atrial fibrillation; myocardial infraction; pneumonia; pneumothorax; hemothorax; prolonged air leak (PAL); and acute respiratory distress syndrome (ARDS). Pneumonia was defined by the need for antibiotics for a suspected chest infection based on lung opacities, fever, or an elevated white blood cell count $>12,000$ per $\mu \mathrm{L}$. PAL was defined as an air leak lasting beyond postoperative day 7 .

\section{Statistical analysis}

The baseline characteristics and clinical outcomes were described using numbers and percentages for categorical variables and median and range for continuous measurements. Comparison of peri- and post-operative variables between complex and simple segmentectomy were analyzed with Fisher's exact test for categorical variables, and the Mann-Whitney $U$ test for continuous variables. Univariate logistic regression was used to explore the association between patient characteristics and complications or delayed discharge [ $>5$ days of length of stay (LOS)]. Odds ratio (OR) with $95 \%$ confidence intervals (CI) were reported. A $\mathrm{P}$ value less than 0.05 was considered statistically significant. All analyses were performed using STATA software, version 14 (StataCorp LLC, Texas, USA).

\section{Results}

A total of 232 patients (114 men; median age 67 years; range, 29-87 years) underwent VATS anatomical pulmonary segmentectomy. CS were realized in 111 patients and SS in 121 patients. Table 1 summarizes the patient characteristics for both groups. The groups were not perfectly matched with slightly younger patients in the CS group and fewer comorbidities. In addition, the CS group patients presented statistically better pulmonary function with higher preoperative forced expiratory volume in 1 second (FEV1) and diffusing capacity of lung for carbon monoxide (DLCO).

Perioperative and postoperative outcomes are summarized in Table 2. Primary lung cancer was the main indication for surgery in both groups with $71 \%$ in CS and $81 \%$ in SS groups. Adenocarcinoma was the most frequent type of pulmonary cancer in both groups. Definitive histological diagnosis demonstrated early stage cancer in most patients in (pT1 in $82 \%$ vs. $78 \%$ ) both groups and an overall nodal upstaging of $8.5 \%$. Final pathological diagnosis showed tumor $>2 \mathrm{~cm}$ in 23 patients in the SS group and 18 patients in the CS group. In the CS groups, the segmentectomy was performed more frequently on the right side and on the upper lobe compared with the SS group. Multiple segments were more frequently removed in the SS group. The operative time were equivalent between both groups. Conversion to open thoracotomy was necessary in 9 patients $(3.9 \%)$ : CS group $(n=4)$ for hemorrhage $(\mathrm{n}=2)$, calcified lymph node $(\mathrm{n}=1)$ and dense pleural adhesions $(\mathrm{n}=1)$; SS group $(\mathrm{n}=5)$ for hemorrhage $(\mathrm{n}=1)$, calcified lymph node $(\mathrm{n}=3)$ and dense pleural adhesion $(\mathrm{n}=1)$. Interestingly, CS were more frequently realized 2 years after implementation of the VATS segmentectomy program with only $23 \%$ of cases performed during the first 2 years. The overall 30 -day mortality and morbidity rates was $0.8 \%$ and $29.7 \%$, respectively (Table 3). Two patients died in the CS group from severe ARDS on post-operative day 3 and 6 respectively, not related to the surgical status. The post-operative complication rates were similar in both groups ( $30 \%$ vs. $30 \%$ respectively, $\mathrm{P}=0.99)$. The overall reoperation rate was $4.7 \%$. In the CS group, re-operation 
Table 1 Patients characteristics

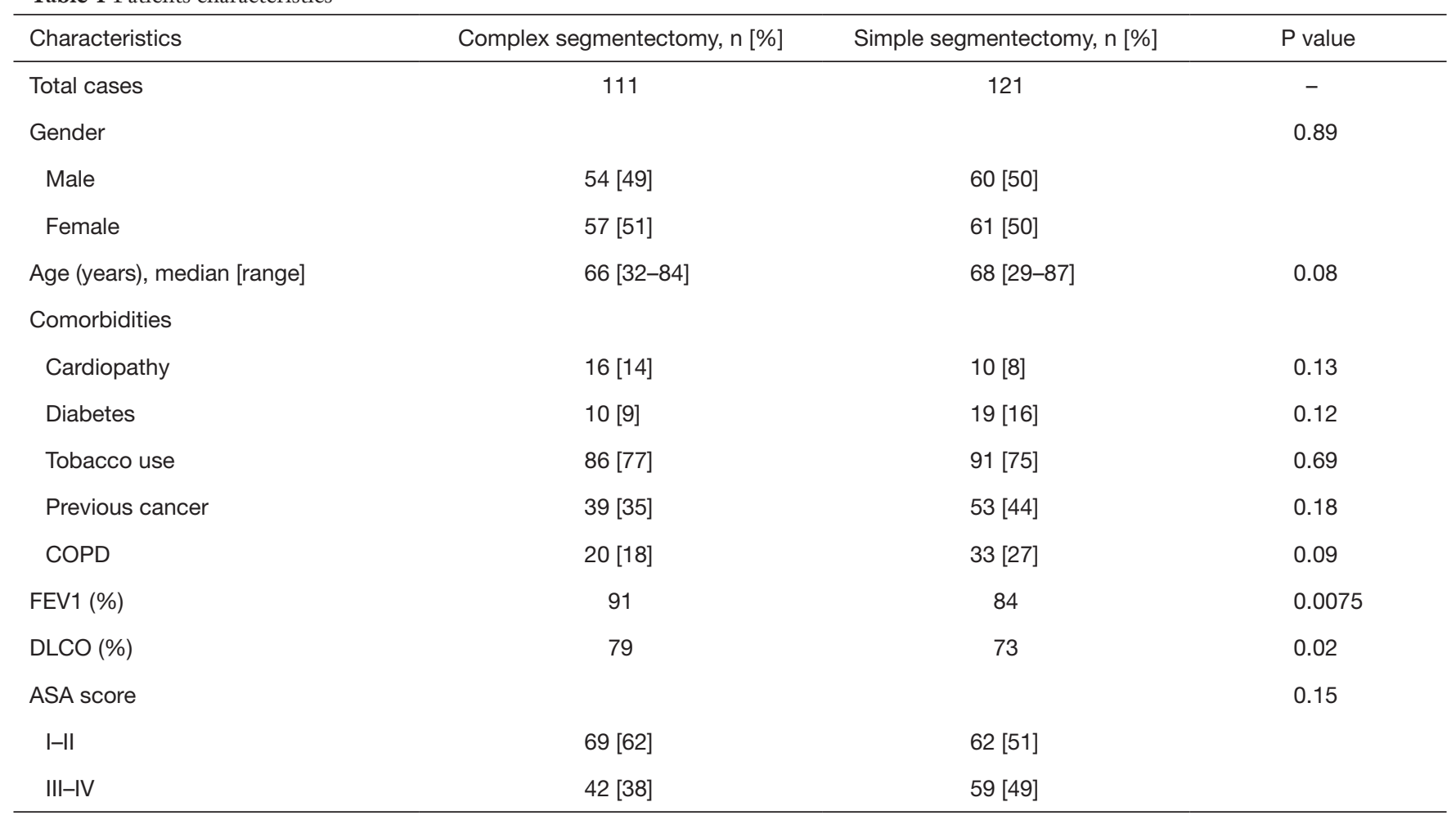

COPD, chronic obstructive pulmonary disease; FEV1, forced expiratory volume in 1 second; DLCO, diffusing capacity of the lung for carbon monoxide; ASA, American Society of Anesthesiologists.

Table 2 Surgical characteristics for complex and simple VATS segmentectomies

\begin{tabular}{|c|c|c|c|}
\hline Characteristics & Complex segmentectomy, n [\%] & Simple segmentectomy, $\mathrm{n}$ [\%] & $P$ value \\
\hline Indications & & & 0.08 \\
\hline Lung cancer & 79 [71] & 98 [81] & \\
\hline Metastasis & $12[11]$ & 14 [12] & \\
\hline Pathology & & & 0.79 \\
\hline Adenocarcinoma & $60 / 79[76]$ & $72 / 98[73]$ & \\
\hline Squamous cells carcinoma & $14 / 79[18]$ & $15 / 98[15]$ & \\
\hline Others & $5 / 79[6]$ & $11 / 98[11]$ & \\
\hline
\end{tabular}

Table 2 (continued) 
Table 2 (continued)

\begin{tabular}{|c|c|c|c|}
\hline Characteristics & Complex segmentectomy, $\mathrm{n}$ [\%] & Simple segmentectomy, n [\%] & $P$ value \\
\hline pT1 & $65[82]$ & 76 [78] & \\
\hline рT2 & $11[14]$ & $18[18]$ & \\
\hline pT3 & $2[3]$ & $4[4]$ & \\
\hline \multicolumn{4}{|l|}{ pN stage } \\
\hline pNO & 72 [91] & 90 [92] & 0.88 \\
\hline $\mathrm{pN} 1$ & $2[3]$ & $5[5]$ & \\
\hline pN2 & $5[6]$ & $3[3]$ & \\
\hline Lower lobe & 27 [24] & 72 [60] & \\
\hline Multiple segment & $44[40]$ & $65[54]$ & 0.03 \\
\hline Side & & & 0.0004 \\
\hline Right & $53[48]$ & $31[26]$ & \\
\hline Left & $58[52]$ & 90 [74] & \\
\hline Segment & & & - \\
\hline S1 & $19[17]$ & - & \\
\hline S2 & 13 [12] & - & \\
\hline S6 & - & $56[46]$ & \\
\hline Basilar (S7-8-9-10) & - & 16 [13] & \\
\hline S8 & $12[11]$ & - & \\
\hline S9 & $5[5]$ & - & \\
\hline S10 & $6[5]$ & - & \\
\hline S8-9 & $1[1]$ & - & \\
\hline S9-10 & $3[3]$ & - & \\
\hline Operative time (mean) (minutes) & 145 & 143 & 0.79 \\
\hline Operation performed within the first 2 years & 25 [23] & 46 [38] & 0.01 \\
\hline
\end{tabular}

VATS, video-assisted thoracic surgery; S, segment. 
Table 3 Post-operative outcomes

\begin{tabular}{lccc}
\hline Outcomes & Complex segmentectomy, $\mathrm{n}[\%]$ & Simple segmentectomy, $\mathrm{n}$ [\%] & $\mathrm{P}$ value \\
\hline Total cases & 111 & 121 & - \\
Mortality & $2[2]$ & 0 & 0.14 \\
Complications & & & 0.99 \\
Overall & $33[30]$ & $36[30]$ & 0.65 \\
Pulmonary & $27[24]$ & $33[27]$ & 0.064 \\
Pneumonia & $8[7]$ & $18[15]$ & 1 \\
Pulmonary embolism & 0 & $1[1]$ & 0.37 \\
Air leak & $12[11]$ & $9[7]$ & 0.51 \\
Empyema & $2[2]$ & $1[1]$ & 0.51 \\
Hemothorax & $2[2]$ & $1[1]$ & 0.66 \\
Pneumothorax & $8[7]$ & $7[6]$ & 0.66 \\
Atrial fibrillation & $5[5]$ & $7[6]$ & 0.93 \\
Renal failure & $2[2]$ & $2[2]$ & 0.51 \\
Urinary retention & $2[2]$ & $1[1]$ & 1 \\
Digestive hemorrhage & 0 & $1[1]$ & 0.76 \\
Re-operation & $5[4.5]$ & $6[5]$ & 0.61 \\
Re-admission & $1[1]$ & $2[2]$ & 0.95 \\
Length of drainage (days), median [range] & $1[0-33]$ & $2[1-19]$ & 0.026 \\
Length of stay (days), median [range] & $5[1-36]$ & $7[2-31]$ & 0.0018 \\
Delayed discharge (>5 days) & $49[44]$ & $764]$ &
\end{tabular}

was necessary for empyema ( $\mathrm{n}=2)$, PAL $(\mathrm{n}=1)$, hemothorax $(\mathrm{n}=1)$ and completion lobectomy for nodal infiltration $(\mathrm{n}=1)$. In the SS group, re-operation was realized for PAL $(n=2)$, hemothorax $(\mathrm{n}=1)$, massive subcutaneous emphysema $(\mathrm{n}=1)$, lingular torsion $(\mathrm{n}=1)$ and completion lobectomy for $\mathrm{R} 1$ resection on the stapler line. Median length of drainage was not statistically different among both groups. However, CS group presented a shorter LOS in comparison to simple segmentectomy. During the first 2 years post-operatively, the CS group did not show a statistical difference in terms of total complications (OR 0.69; 95\% CI: 0.24-1.92; $\mathrm{P}=0.47$ ) or delayed discharge $>5$ days (OR 0.99; $95 \%$ CI: $0.40-2.40 ; \mathrm{P}=0.98$ ).

On univariate analysis (Table 4), the American Society of Anesthesiologists (ASA) score $>2$ and tobacco use were significantly associated with postoperative complications. Right-sided segmentectomies presented significantly fewer post-operative complications. As shown in Table 5, significant risk factors for delayed discharge ( $>5$ days of LOS) were identified as chronic obstructive pulmonary disease (COPD), tobacco use, FEV1 $<60$, DLCO $<60$, and removal of multiple segments. On the other hand, rightsided procedures and CS were associated with a decreased rate of delayed discharge.

\section{Discussion}

Our study focused on patients undergoing segmentectomies by VATS. We have recently published our preliminary experience after implementation of a segmentectomy program showing that segmentectomies by VATS can be realized safely with acceptable morbidity and low mortality even during an initial experience (8). In this multicenter study, which compared surgical outcomes between SS and CS by VATS, we have shown that complications were comparable between the two groups, and that CS were 
Table 4 Univariate analysis for risk factors of post-operative complication after VATS segmentectomies

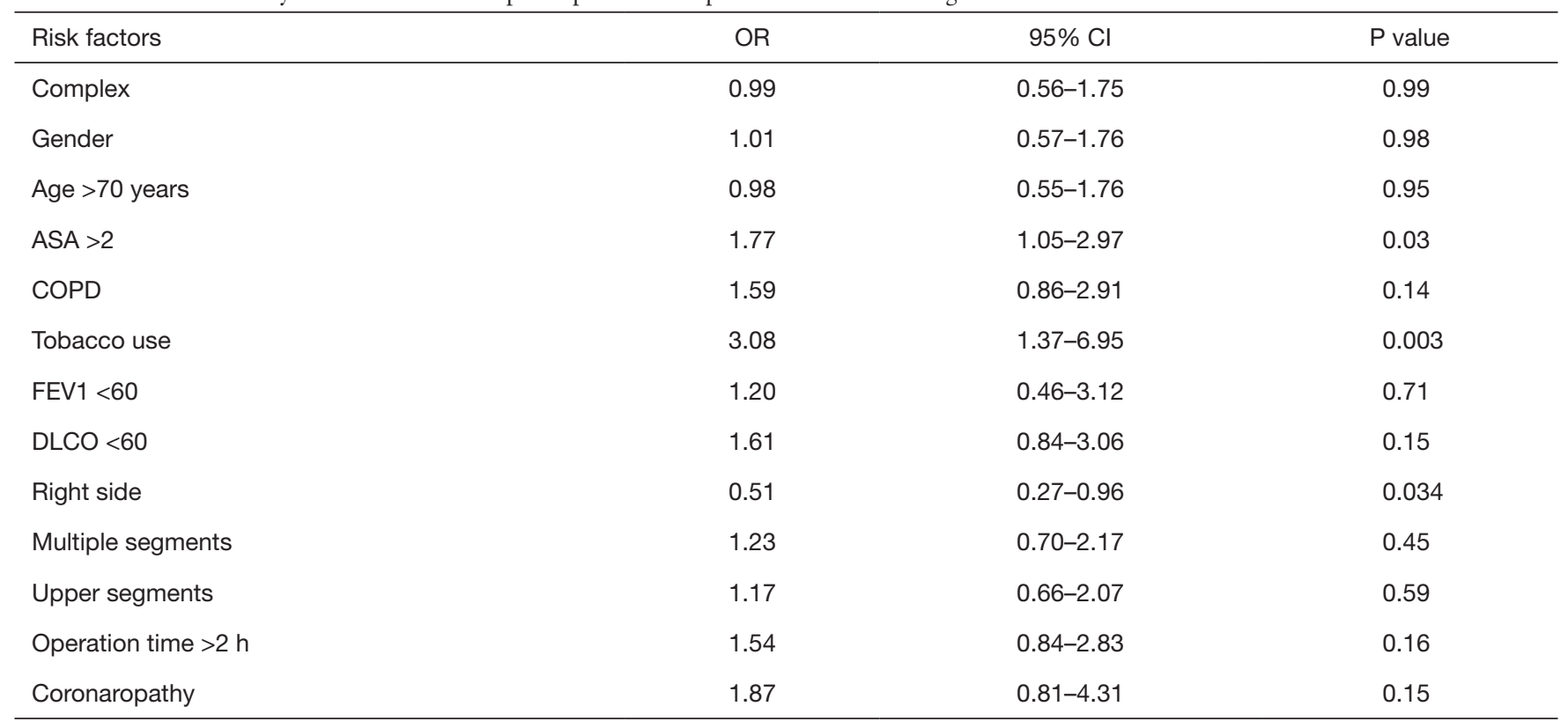

VATS, video-assisted thoracic surgery; OR, odds ratio; CI, confidence interval; ASA, American Society of Anesthesiologists; COPD, chronic obstructive pulmonary disease; FEV1, forced expiratory volume in 1 second; DLCO, diffusing capacity of the lung for carbon monoxide.

Table 5 Univariate analysis for risk factors of delayed discharge ( $>5$ days) after VATS segmentectomies

\begin{tabular}{llll}
\hline Risk factors & OR & $95 \% \mathrm{Cl}$ & P value \\
\hline Complex & 0.43 & $0.25-0.73$ & 0.0018 \\
Gender (male) & 0.63 & $0.37-1.07$ & 0.09 \\
Age $>$ 70 years & 1.66 & $0.97-2.86$ & 0.0625 \\
ASA $>2$ & 1.88 & $1.15-3.08$ & 0.098 \\
COPD & 2.57 & $1.39-4.75$ & 0.0019 \\
Tobacco use & 1.98 & $1.07-3.66$ & 0.03 \\
FEV1 $<60$ & 3.90 & $1.27-11.99$ & 0.0084 \\
DLCO $<60$ & 2.04 & $1.07-3.91$ & 0.0265 \\
Right side & 0.51 & $0.29-0.87$ & 0.0137 \\
Multiple segments & 2.08 & $1.22-3.52$ & 0.0061 \\
Upper segments & 1.08 & $0.64-1.82$ & 0.77 \\
Operation time $>2 \mathrm{~h}$ & 1.51 & $0.87-2.58$ & 0.135 \\
Coronaropathy & 1.14 & $0.50-2.61$ & 0.74 \\
\hline VATS, video-assisted thoraci & & \\
\hline
\end{tabular}

VATS, video-assisted thoracic surgery; OR, odds ratio; Cl, confidence interval; ASA, American Society of Anesthesiologists; COPD, chronic obstructive pulmonary disease; FEV1, forced expiratory volume in 1 second; DLCO, diffusing capacity of the lung for carbon monoxide. 
performed more frequently after an initial experience of 2 years in anatomical resection by VATS.

Complex VATS segmentectomy remains less frequently performed due to the complexity of the procedure. We therefore thought it was important to analyze the rate of post-operative complications, especially during the learning curve of this procedure. We decided to analyze peri- and post-operative end-points for patients undergoing VATS segmentectomy, and stratify them by procedure (simple $v s$. complex). Whilst there could have been other possible approaches, this one had the advantage of analyzing endpoints of direct interest to the surgeon and the patient, and to focus on clinically relevant questions. Our study involved a large enough group of patients, separated in sub-groups of approximately the same size, to draw initial conclusions with some degree of statistical soundness, despite the slight imperfections in the matching of patients between the two groups in terms of age, comorbidities and pre-operative pulmonary function.

We could make several observations. First, laterality of the surgical intervention was different in the two groups: patients undergoing CS were more frequently operated on the right lung and in the upper lobe than the patients undergoing a SS. These results could be explained by the fact that on the left side, trisegmentectomy (upper division segmentectomy, lingular sparing lobectomy) of the upper lobe is currently proposed as alternative to upper lobectomy and is an equivalent on the left side to the right upper lobe. As expected, the patients undergoing a SS were more frequently operated on multiple distant segments than those undergoing a CS. This follows directly from definitions of SS and CS established in our study. Surprisingly, however, we found that despite the increased technical difficulties, CS did not end up being longer operations. This might be explained by the observation that the majority of CS were carried out after the team had gathered more than 2 years of experience with the VATS procedures, thus benefitting from the overall VATS learning curve. Recently, Handa $e t a l$. analyzed the surgical results of complex VATS segmentectomies $(\mathrm{n}=117)$ compared to simple VATS segmentectomies ( $\mathrm{n}=92)$ for NSCLC (10). Interestingly, they reported an increased operating time for CS (180 vs. 143.5 minutes, $\mathrm{P}<0.0001$ ), without an elaborate explanation on this observation. In our group of patients, operative time was similar for both types of segmentectomy even though CS requires deeper dissection of the segmental bronchus, artery, and vein and division of several intersegmental planes. Based on our experience, we find it fair to state that the additional technical difficulties are not reflected by a concomitant longer operative time if the learning curve is already reached for VATS lobectomy and surgeons are experienced with more than 100 VATS lobectomies.

In terms of post-operative results, we found that compared to SS, CS by VATS presented similar overall and pulmonary post-operative complication rates. This is extremely encouraging because this is likely to increase the acceptance of VATS for CS both for surgeons and patients. In addition, we did not observe differences in terms of reoperation rates or re-admission rates, figures that are also likely to bring solace to prospective patients and surgeons who might initially be deterred by the technical difficulties associated with the procedure. In general, these postoperative complication rates are better than previously reported rates, published in reports that found average overall complication rates up to $35 \%$ after segmentectomy (8,11-13). Our univariate analysis of risk factors relevant for post-operative complications included left-sided operation, tobacco use and ASA score $>2$. Some of these risk factors were already identified in other series $(8,13,14)$. In general, tobacco use and ASA score $>2$ seem to be expected risk factors for increased post-operative complications. On the other hand, the fact that right-sided operations are associated with fewer post-operative complications is not fully explained and might be due to some bias. One might hypothesize that segmentectomy of the right lung may be better tolerated due to the size of the lung.

We were surprised to observe a statistical difference for the LOS with a shorter LOS in favor of CS, whereas median length of drainage was not statistically different. We also observed that some risk factors were correlated with a longer LOS (COPD; tobacco use; FEV1 <60; DLCO <60; operation on multiple segments), none of which seems to be particularly surprising. On the other hand, the fact that CS were associated with decreased rate of delayed discharge might come as a surprise. We think that these results could be explained by two aspects: first, most CS were realized after 2 years of experience in VATS segmentectomy and thus the learning curve was progressively reached allowing comparable length of operative time and post-operative outcomes. On the other hand, an enhanced recovery after surgery (ERAS) program has been introduced in one of the Hospitals which allowed to reduce the LOS from 7 to 4 days in the last 2 years for anatomical lung resections by VATS (15). In their study, Handa et al. reported overall complications $(24.8 \%$ vs. $22.8 \%$ ), and PAL (11.9\% vs. $10.9 \%)$ nearly equivalent for both groups and in the 
same range as the rates we report (10). They also found a decreased LOS (7 vs. 6 days) and decreased length of drainage (3vs. 2 days) in favor of the SS without statistical significance. This might be seen as an opposite trend to the results that we report, although their results (as ours) point to differences of small magnitude, which might well explain the discordant directions.

In case of NSCLC, the location of the tumor and the localization of the appropriate intersegmental plane is determinant to achieve an oncological safety margin. In almost one third of cases, we used ICG as described in previous work, mainly during the initial phase $(16,17)$. We consider that the use of intravenous ICG allows precise anatomical resection and facilitates the surgical approach during VATS to improve the success and quality of the segmentectomy at least during the learning curve. The success rate of ICG identification of the intersegmental plane has been reported to range from $88 \%$ to $100 \%$. In addition, it has been shown that $10 \%$ of patients had modified intersegmental plane resection avoiding potential post-operative complication such as infarction or infection (17). These observations are generally positive but should not obliterate the fact that pulmonary segmentectomy in NSCLC patients remains controversial until the definitive results of the two ongoing randomized trials (NCT00499330) and (JCOG0802/WJOG4607L) comparing VATS segmentectomy and lobectomy. Pulmonary segmentectomy is currently considered appropriate for tumors with a diameter $\leq 2 \mathrm{~cm}$ with no nodal involvement but segmentectomy may offer better oncological outcomes than wedge resection in cases of early stage NSCLC by providing wider and deeper surgical margins and better nodal evaluation. Nodal upstaging was $8.5 \%$ which is well comparable with other surgical series with small tumors (18). Only one patient presented an R1 resection not seen on frozen section but on definitive pathology thus required secondary lobectomy. We are currently evaluating the long-term survival and local recurrence rates, but due to the short follow-up, this point was not analyzed in the present study.

Our retrospective study presents several limitations. First, it includes a small number of patients, treated in two different institutions. This was a retrospective study, and we cannot exclude the possibility of a selection bias. Due to the retrospective nature of the study, we were not able to determine if there have been patients who were scheduled for an intentional segmentectomy but finally underwent lobectomy as a result of an intra-operative decision and thus fell out of the current analysis. In addition, one institution had an ERAS program in place and the other one did not. This means that the group of patients studied was inhomogeneous in several respects and that further stratification came at the expense of statistical power. Second, because of its retrospective nature, our study could not include data about complications during the operation, thus possibly missing some relevant information. We believe that complex VATS segmentectomy will only gain full acceptance when the above points have been successfully addressed and long enough follow-up has yielded convincing oncological results. In this respect, our study should be seen as an initial step to expand the reach of VATS for increasingly complex surgical interventions.

In conclusion, our retrospective study of experience in two institutions suggests that despite theoretical major difficulties of CS, post-operative outcomes are similar to those of simple segmentectomy. Gradual experience and progressive acceptance seem to be key elements for successful implementation and could explain shorter LOS.

\section{Acknowledgments}

We would like to thank Audrey Roth for the data management and the collection and extraction of the data.

\section{Footnote}

Conflicts of Interest: Presented as poster at the 27th Annual Meeting of the European Society of Thoracic Surgery, Dublin, Slovenia, 9-12 June 2019.

Ethical Statement: The authors are accountable for all aspects of the work in ensuring that questions related to the accuracy or integrity of any part of the work are appropriately investigated and resolved. The Local Ethics Committee approved the study and individual consent was waived (Referral number: 2018-00179).

\section{References}

1. Villamizar N, Swanson SJ. Lobectomy vs. segmentectomy for NSCLC $(\mathrm{T}<2 \mathrm{~cm})$. Ann Cardiothorac Surg 2014;3:160-6.

2. Razi SS, John MM, Sainathan S, et al. Sublobar resection is equivalent to lobectomy for T1a non-small cell lung cancer in the elderly: a Surveillance, Epidemiology, and End Results database analysis. J Surg Res 2016;200:683-9. 
3. Koike T, Kitahara A, Sato S, et al. Lobectomy Versus Segmentectomy in Radiologically Pure Solid SmallSized Non-Small Cell Lung Cancer. Ann Thorac Surg 2016;101:1354-60.

4. Song CY, Sakai T, Kimura D, et al. Comparison of perioperative and oncological outcomes between videoassisted segmentectomy and lobectomy for patients with clinical stage IA non-small cell lung cancer: a propensity score matching study. J Thorac Dis 2018;10:4891-901.

5. Landreneau RJ, Normolle DP, Christie NA, et al. Recurrence and survival outcomes after anatomic segmentectomy versus lobectomy for clinical stage I nonsmall-cell lung cancer: a propensity-matched analysis. J Clin Oncol 2014;32:2449-55.

6. Altorki NK, Yip R, Hanaoka T, et al. Sublobar resection is equivalent to lobectomy for clinical stage $1 \mathrm{~A}$ lung cancer in solid nodules. J Thorac Cardiovasc Surg 2014;147:75462; Discussion 762-4.

7. Karenovics W, Gonzalez M. How to decrease technical obstacles to difficult video-assisted thoracoscopic surgery segmentectomy? J Thorac Dis 2019;11:53-6.

8. Bedat B, Abdelnour-Berchtold E, Krueger T, et al. Clinical outcome and risk factors for complications after pulmonary segmentectomy by video-assisted thoracoscopic surgery: results of an initial experience. J Thorac Dis 2018;10:5023-9.

9. Nakazawa S, Shimizu K, Mogi A, et al. VATS segmentectomy: past, present, and future. Gen Thorac Cardiovasc Surg 2018;66:81-90.

10. Handa Y, Tsutani Y, Mimae T, et al. Surgical Outcomes of Complex Versus Simple Segmentectomy for Stage I Non-Small Cell Lung Cancer. Ann Thorac Surg 2019;107:1032-9.

11. Traibi A, Grigoroiu M, Boulitrop C, et al. Predictive

Cite this article as: Bédat B, Abdelnour-Berchtold E, Krueger T, Perentes JY, Zellweger M, Triponez F, Karenovics W, Gonzalez M. Impact of complex segmentectomies by videoassisted thoracic surgery on peri-operative outcomes. J Thorac Dis 2019;11(10):4109-4118. doi: 10.21037/jtd.2019.10.07 factors for complications of anatomical pulmonary segmentectomies. Interact Cardiovasc Thorac Surg 2013;17:838-44.

12. Deng B, Cassivi SD, de Andrade M, et al. Clinical outcomes and changes in lung function after segmentectomy versus lobectomy for lung cancer cases. J Thorac Cardiovasc Surg 2014;148:1186-1192.e3.

13. Smith CB, Kale M, Mhango G, et al. Comparative outcomes of elderly stage I lung cancer patients treated with segmentectomy via video-assisted thoracoscopic surgery versus open resection. J Thorac Oncol 2014;9:383-9.

14. Licker M, Karenovics W, Diaper J, et al. Short-Term Preoperative High-Intensity Interval Training in Patients Awaiting Lung Cancer Surgery: A Randomized Controlled Trial. J Thorac Oncol 2017;12:323-33.

15. Gonzalez M, Abdelnour-Berchtold E, Perentes JY, et al. An enhanced recovery after surgery program for videoassisted thoracoscopic surgery anatomical lung resections is cost-effective. J Thorac Dis 2018;10:5879-88.

16. Guigard S, Triponez F, Bedat B, et al. Usefulness of nearinfrared angiography for identifying the intersegmental plane and vascular supply during video-assisted thoracoscopic segmentectomy. Interact Cardiovasc Thorac Surg 2017;25:703-9.

17. Bedat B, Triponez F, Sadowski SM, et al. Impact of nearinfrared angiography on the quality of anatomical resection during video-assisted thoracic surgery segmentectomy. J Thorac Dis 2018;10:S1229-34.

18. Stiles BM, Kamel MK, Nasar A, et al. The importance of lymph node dissection accompanying wedge resection for clinical stage IA lung cancer. Eur J Cardiothorac Surg 2017;51:511-7. 
Table S1 STROBE Statement—checklist of items that should be included in reports of observational studies

\begin{tabular}{|c|c|c|c|}
\hline Item & Item No. & Recommendation & Page \\
\hline \multirow[t]{2}{*}{ Title and abstract } & 1 & (a) Indicate the study's design with a commonly used term in the title or the abstract & 1 \\
\hline & & (b) Provide in the abstract an informative and balanced summary of what was done and what was found & 1 \\
\hline \multicolumn{4}{|l|}{ Introduction } \\
\hline Background/rationale & 2 & Explain the scientific background and rationale for the investigation being reported & 2 \\
\hline Objectives & 3 & State specific objectives, including any prespecified hypotheses & 2 \\
\hline \multicolumn{4}{|l|}{ Methods } \\
\hline Study design & 4 & Present key elements of study design early in the paper & 2 \\
\hline Setting & 5 & Describe the setting, locations, and relevant dates, including periods of recruitment, exposure, follow-up, and data collection & $2-3$ \\
\hline \multirow[t]{5}{*}{ Participants } & 6 & (a) Cohort study-Give the eligibility criteria, and the sources and methods of selection of participants. Describe methods of follow-up & $2-3$ \\
\hline & & $\begin{array}{l}\text { Case-control study-Give the eligibility criteria, and the sources and methods of case ascertainment and control selection. Give the rationale for the } \\
\text { choice of cases and controls }\end{array}$ & \\
\hline & & Cross-sectional study-Give the eligibility criteria, and the sources and methods of selection of participants & \\
\hline & & (b) Cohort study -For matched studies, give matching criteria and number of exposed and unexposed & $2-3$ \\
\hline & & Case-control study-For matched studies, give matching criteria and the number of controls per case & \\
\hline Variables & 7 & Clearly define all outcomes, exposures, predictors, potential confounders, and effect modifiers. Give diagnostic criteria, if applicable & 3 \\
\hline Data sources/measurement & $8^{*}$ & $\begin{array}{l}\text { For each variable of interest, give sources of data and details of methods of assessment (measurement). Describe comparability of assessment methods if } \\
\text { there is more than one group }\end{array}$ & 3 \\
\hline Bias & 9 & Describe any efforts to address potential sources of bias & 3 \\
\hline Study size & 10 & Explain how the study size was arrived at & $2-3$ \\
\hline Quantitative variables & 11 & Explain how quantitative variables were handled in the analyses. If applicable, describe which groupings were chosen and why & 3 \\
\hline \multirow[t]{7}{*}{ Statistical methods } & 12 & (a) Describe all statistical methods, including those used to control for confounding & 3 \\
\hline & & (b) Describe any methods used to examine subgroups and interactions & 3 \\
\hline & & (c) Explain how missing data were addressed & N/A \\
\hline & & (d) Cohort study - If applicable, explain how loss to follow-up was addressed & 3 \\
\hline & & Case-control study-If applicable, explain how matching of cases and controls was addressed & \\
\hline & & Cross-sectional study - If applicable, describe analytical methods taking account of sampling strategy & \\
\hline & & (e) Describe any sensitivity analyses & N/A \\
\hline \multicolumn{4}{|l|}{ Results } \\
\hline \multirow[t]{3}{*}{ Participants } & $13^{*}$ & $\begin{array}{l}\text { (a) Report numbers of individuals at each stage of study-e.g., numbers potentially eligible, examined for eligibility, confirmed eligible, included in the } \\
\text { study, completing follow-up, and analysed }\end{array}$ & $3-4$ \\
\hline & & (b) Give reasons for non-participation at each stage & $3-4$ \\
\hline & & (c) Consider use of a flow diagram & Table 1 \\
\hline \multirow[t]{3}{*}{ Descriptive data } & $14^{*}$ & (a) Give characteristics of study participants (e.g., demographic, clinical, social) and information on exposures and potential confounders & $3-4$ \\
\hline & & (b) Indicate number of participants with missing data for each variable of interest & $3-4$ \\
\hline & & (c) Cohort study-Summarise follow-up time (e.g., average and total amount) & N/A \\
\hline \multirow[t]{3}{*}{ Outcome data } & $15^{*}$ & Cohort study-Report numbers of outcome events or summary measures over time & $3-4$ \\
\hline & & Case-control study-Report numbers in each exposure category, or summary measures of exposure & \\
\hline & & Cross-sectional study-Report numbers of outcome events or summary measures & \\
\hline \multirow[t]{3}{*}{ Main results } & 16 & $\begin{array}{l}\text { (a) Give unadjusted estimates and, if applicable, confounder-adjusted estimates and their precision (e.g., } 95 \% \text { confidence interval). Make clear which } \\
\text { confounders were adjusted for and why they were included }\end{array}$ & 7 \\
\hline & & (b) Report category boundaries when continuous variables were categorized & \\
\hline & & (c) If relevant, consider translating estimates of relative risk into absolute risk for a meaningful time period & \\
\hline Other analyses & 17 & Report other analyses done-e.g., analyses of subgroups and interactions, and sensitivity analyses & N/A \\
\hline \multicolumn{4}{|l|}{ Discussion } \\
\hline Key results & 18 & Summarise key results with reference to study objectives & $6-9$ \\
\hline Limitations & 19 & Discuss limitations of the study, taking into account sources of potential bias or imprecision. Discuss both direction and magnitude of any potential bias & 9 \\
\hline Interpretation & 20 & $\begin{array}{l}\text { Give a cautious overall interpretation of results considering objectives, limitations, multiplicity of analyses, results from similar studies, and other relevant } \\
\text { evidence }\end{array}$ & 9 \\
\hline Generalizability & 21 & Discuss the generalizability (external validity) of the study results & 9 \\
\hline \multicolumn{4}{|l|}{ Other information } \\
\hline Funding & 22 & Give the source of funding and the role of the funders for the present study and, if applicable, for the original study on which the present article is based & 9 \\
\hline
\end{tabular}

